\title{
Receptor Tyrosine Kinase Targeting in Multicellular Spheroids
}

\author{
Susan Breslin and Lorraine 0’Driscoll
}

\begin{abstract}
While growing cells as a monolayer is the traditional method for cell culture, the incorporation of multicellular spheroids into experimental design is becoming increasingly popular. This is due to the understanding that cells grown as spheroids tend to replicate the in vivo situation more reliably than monolayer cells. Thus, the use of multicellular spheroids may be more clinically relevant than monolayer cell cultures. Here, we describe methods for multicellular 3D spheroid generation that may be used to provide samples for receptor tyrosine kinase (and other protein) detection. Methods described include the forced-floating poly-HEMA method, the hanging-drop method, and the use of ECM to form multicellular 3D spheroids.
\end{abstract}

Key words Monolayer, 3D spheroid, Poly-HEMA, Hanging-drop, ECM

\section{Introduction}

Monolayer (2D) cell culture involves the growth of cells attached flat to the surface of cell culture plastic and is the traditional method for growing cells in the laboratory. $2 \mathrm{D}$ cell culture has provided a means for investigating in vitro cell biology, since its introduction in 1907 [1]. In vitro cell culture has enabled scientists to investigate biological processes outside of the in vivo environment, which is costly and can be complicated by ethics associated with the use of animals for research.

Although 2D cell culture has contributed massively to furthering our knowledge of cell biology and its related fields, it does not reflect the way cells grow and interact with each other in the in vivo environment, where cells grow in 3D. The difference in spatial arrangement of cells in 2D compared to 3D results in differences in cell signalling. Differences in cellular arrangement between cells grown in 2D and 3D cultures can be seen in Fig. 1. Cell signalling is at the core of how a cell behaves and as such, alterations in signals between cells grown in $2 \mathrm{D}$ and $3 \mathrm{D}$ can result in alterations in cell proliferation, differentiation and numerous other cellular functions $[2,3]$. 


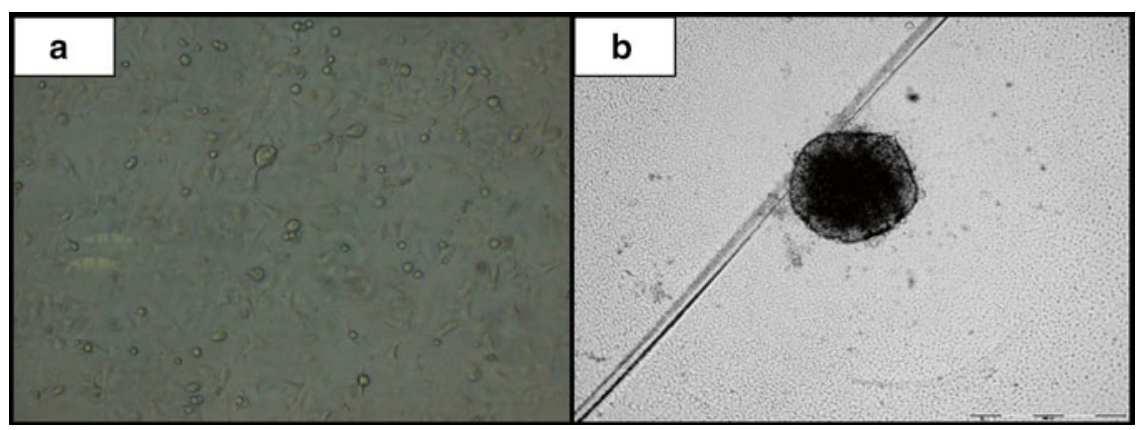

Fig. 12 D and 3D cell cultures. (a) HCC1954 breast cancer cells grown flat in 2D. (b) HCC1954 cells grown in 3D using the poly-HEMA method

Signalling in cells grown in $3 \mathrm{D}$ appears to replicate the in vivo situation more accurately than $2 \mathrm{D}$ and so it has been proposed that 3D multicellular spheroid culture should be incorporated into research more extensively, for more reliable information.

Receptor tyrosine kinase (RTK) expression has been reported to be expressed differently in cells when grown 2D and 3D. For example, levels of RTKs VEGFR-1, VEGFR-2, TIE-1, and TIE-2 were found to be increased in HUVEC cells grown in $3 \mathrm{D}$ compared to those grown in 2D cultures [4]. Additionally, Pickl and Ries [5] reported that EGFR and HER3 expression was decreased in SKBR3 cells grown as 3D spheroids compared to 2D, whereas HER2 expression was increased in SKOV-3 cells as 3D spheroids compared to cells grown as $2 \mathrm{D}$ cultures. The effect of cell culture dimension on expression of receptors is evident and highlights the importance of incorporating 3D cell culture into experimental designs in order to obtain the most biologically relevant information from in vitro data when targeting RTKs and other proteins.

In vitro $3 \mathrm{D}$ cell culture can be performed by many methods, which have been reviewed in Breslin and O'Driscoll [6]; some more commonly used methods will be detailed here. (1) The forced-floating method of 3D cell culture involves coating cell culture plastic with a substance, e.g., poly-2-hydroxyethyl methacrylate (poly-HEMA), that prevents cell adherence prior to cell seeding [7]. (2) The hanging-drop method involves hanging drops of cells in suspension [8]. (3) The use of scaffolds [9] or matrices [10] enable 3D formation while providing extracellular support. (4) Agitation-based approaches, which use rotary vessels to prevent cell adherence to the culture vessel due to its constant movement [11] or spinner flasks with an internal stirring bar that keep cells and culture medium in constant, gentle motion [12]. All methods for multicellular spheroid generation have advantages and disadvantages. The methods described below are used for their advantage of being able to determine the number of cells seeded per well. 


\section{Materials}

2.1 Cell Culture

2.2 Poly-

HEMA Method

\subsection{Hanging- \\ Drop Method}

2.4 ECM Method

2.5 Spheroid

Pelleting
1. HCC1954 breast cancer cell line (American Type Culture Collection, Rockville, MD, USA).

2. Cell culture medium: RPMI medium with $10 \% \mathrm{FBS}$ and $1 \mathrm{mM}$ L-glutamine.

3. Trypsin-EDTA solution: $0.25 \%$ Trypsin and $0.53 \mathrm{mM}$ EDTA.

4. Phosphate-buffered saline (PBS).

5. Tissue culture-grade vented flasks (e.g., $T 75 \mathrm{~cm}^{2}$ ).

1. $95 \%$ ethanol solution: $95 \mathrm{~mL}$ ethanol and $5 \mathrm{~mL}$ deionised $\mathrm{H}_{2} \mathrm{O}$.

2. Poly-HEMA (Sigma-Aldrich, St Louis, MO, USA): $12 \mathrm{mg} /$ $\mathrm{mL}$ solution. Add $1.2 \mathrm{~g}$ of poly-HEMA to $100 \mathrm{~mL} 95 \%$ ethanol.

3. 96-Well round-bottomed plates.

4. Benchtop centrifuge with plate rotor.

5. Benchtop rocker.

6. Hemocytometer.

1. 96-Well flat-bottomed plates.

1. 96-Well flat-bottomed plates.

2. Extracellular matrix gel (Sigma-Aldrich).

1. Phosphate-buffered saline (PBS).

2. 1 \% BSA solution: $0.1 \mathrm{~g}$ BSA in $100 \mathrm{~mL}$ PBS.

3. $1 \mathrm{~mL}$ pipette tips.

4. 6-Well plates.

\section{Methods}

3.1 Poly-HEMA

Method for Multicellular Spheroids
Coating round-bottom 96-well plates with poly-HEMA prevents cellular adhesion to the cell culture plastic, forcing cells to grow in suspension. This, in turn, encourages them to adhere to each other instead of cell culture vessel in the form of a spheroid. A centrifugation step is included to bring all of the cells seeded to the bottom of each well in order to aid the aggregation process of spheroid formation.

1. Prepare a poly-HEMA solution in a sterile container. Place container on a rocker and allow poly-HEMA to dissolve in solution overnight ( see Note 1). 


\subsection{Hanging-Drop \\ Method for Multicellular Spheroids}

2. Place $50 \mu \mathrm{L}$ of poly-HEMA solution into each well of a 96 -well round-bottomed plate, replace lid leaving it slightly ajar and place at the back of a laminar flow cabinet overnight to allow solution to evaporate.

3. Repeat this process once more and ensure that second coat of poly-HEMA is completely evaporated (see Note 2 ).

4. Grow cells to approximately $70 \%$ confluency in $T 75 \mathrm{~cm}^{2}$ flasks and then prepare for seeding in poly-HEMA coated plates. HCC1954 HER2-overexpressing breast cancer cells are used here as an example ( see Note 3 ). Cells are cleaved from the T75 $\mathrm{cm}^{2}$ culture flask using $3 \mathrm{~mL}$ trypsin, the cell suspension is neutralized with $3 \mathrm{~mL}$ medium and $10 \mu \mathrm{L}$ of this cell suspension is used to count cells with a hemocytometer.

5. Seed cells at a density of $3 \times 10^{3}$ cells $/ 150 \mu \mathrm{L} /$ well in $10 \%$ FBS, 1 \% L-glutamine supplemented RPMI.

6. Plates are then centrifuged at $900 \times g$ for $10 \mathrm{~min}$ to gather cells in the bottom of wells and placed back in incubator at $37^{\circ} \mathrm{C}$ and $5 \% \mathrm{CO}_{2}$.

7. Cells adhere to each other overnight, forming spheroids.

8. Change medium every second day by removing $50 \mu \mathrm{L}$ culture medium and replacing it with $50 \mu \mathrm{L}$ fresh medium (see Note 4 ).

The principle behind the hanging-drop method for multicellular spheroid formation relies on the ability of small drops of fluid being able to stay attached on the underside of a surface due to surface tension. This force can be utilised in cell culture, whereby the fluid involved is a homogeneous cell suspension in culture medium and a drop of this fluid is placed in a sterile flat surface for the formation of multicellular spheroids. Once a cell suspension drop is placed on a surface and that surface is inverted, the drop hangs down but does not detach. Due to gravity, the cells in suspension fall to the bottom of the drop and adhere to each other in the formation of a cellular spheroid.

1. Prepare cells for suspension as in Subheading 3.1, step 4, at a density of 500 cells $/ 25 \mu \mathrm{L} /$ well.

2. Place $25 \mu \mathrm{L}$ of cell suspension in the ring of each well in the lid of a 96-well plate ( see Note 5 ).

3. Once 96 drops have been seeded, in one swift movement turn the lid over.

4. Place $100 \mu \mathrm{L}$ of medium in each well to prevent evaporation of drops, replace the lid and place the plate in an incubator at $37^{\circ} \mathrm{C}$ and $5 \% \mathrm{CO}_{2}$.

5. To exchange medium during $3 \mathrm{D}$ cell culturing with the hanging-drop method, slowly remove $10 \mu \mathrm{L}$ of medium and replace with fresh medium. 


\subsection{ECM Method for Multicellular Spheroids}

\subsection{Harvesting Spheroids for RTK Detection}

3.4.1 Collecting Cells from Poly-HEMA and Hanging-Drop Method
As previously mentioned, cell signaling plays a significant and important role in cell behavior. The loss of cell-ECM signaling is one of the signals limited in 2D culture. It can, therefore, be an advantage of $3 \mathrm{D}$ cell culture that cell-ECM signaling can be reintroduced to in vitro culture, as signaling via cells and ECM can affect the organization of cells and their functions. The ECM method for spheroid formation involves culturing cells on commercially available ECM which comprises proteins commonly derived from the basement membrane, e.g., collagen, growth factors, and laminin.

1. Thaw ECM overnight at $4{ }^{\circ} \mathrm{C}($ see Note 6$)$.

2. Place a 96-well plate in fridge overnight to chill.

3. On day of seeding, place 96 -well plate on ice, pipette $5 \mu \mathrm{L}$ of ECM on base of each well, and spread in an even layer with the tip of pipette.

4. Place plate in an incubator at $37^{\circ} \mathrm{C}$ for approximately $30 \mathrm{~min}$ to allow ECM to begin to set, but not to fully solidify.

5. Prepare cells by detaching from culture flask using $3 \mathrm{~mL}$ tryp$\sin$, neutralize cell suspension with $3 \mathrm{~mL}$ medium. $10 \mu \mathrm{L}$ of this cell suspension is used to count cells with a hemocytometer.

6. Pellet cells by centrifugation at $900 \times g$ for $5 \mathrm{~min}$.

7. Prepare a cell suspension of 1,000 cells $/ 100 \mu \mathrm{L}$ ice-cold medium/well pipette into wells.

8. Place plate in an incubator and allow cells to settle for 15-30 min.

9. Prepare a medium/ECM solution of $10 \mu \mathrm{L}$ ECM and $90 \mu \mathrm{L}$ ice-cold medium and pipette solution down the well walls in order not to disturb seeded cells.

10. Medium/ECM solution can be replaced every other day.

1. Coat $1 \mathrm{~mL}$ pipette tips with $1 \%$ BSA by aspirating and completely dispensing the solution with the tips, then put back the tips in the rack, and allow them to dry before use (see Note 7).

2. Aspirate contents of each well or drop containing spheroids and dispense into a well of a 6-well plate to allow for collection of spheroids into a $1.5 \mathrm{~mL}$ centrifuge tube (Fig. 2).

3 . Once all spheroids are in the 6-well plate, very gently swirl the contents of the wells; this brings the spheroids to the centre of the well.

4. Using a $1 \mathrm{~mL}$ pipette tip, collect and transfer spheroids to a $1.5 \mathrm{~mL}$ tube for washing.

5. Spin tub for $5 \mathrm{~min}$ at $13.2 \times 10^{4} \times g$ at $4{ }^{\circ} \mathrm{C}$.

6 . Remove supernatant without disturbing cell pellet. 
a Poly-HEMA and hanging-drop methods

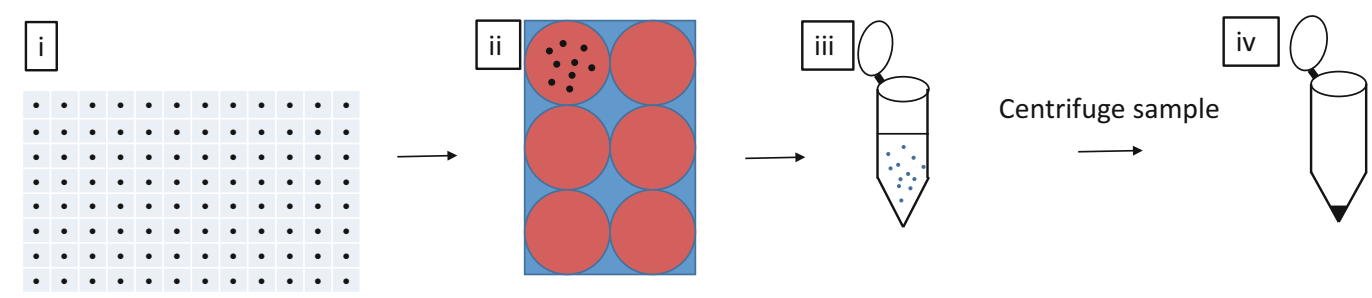

b ECM method
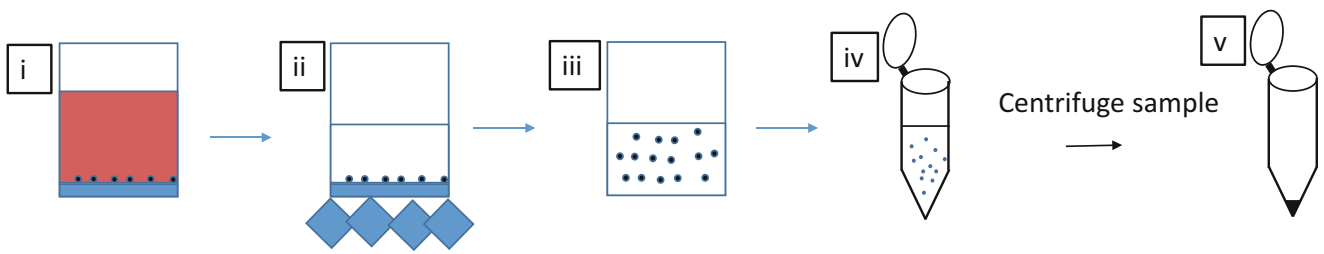

Fig. 2 Sample collection. (a) Collection of 3D cell samples using poly-HEMA and hanging-drop methods. (i-ii) Aspirate spheroids from culture dishes to 6-well plates. (iii) Transfer cells to an Eppendorf for washing. Centrifuge sample, remove supernatant, resuspend in PBS, and centrifuge again. (iv) Remove supernatant. (b) Collection of 3D samples using ECM method. (i) Remove medium, wash cells with PBS $\times 2$, and add $150 \mu \mathrm{L}$ PBS. (ii) On ice, break up cells and ECM in PBS. (iv) Once cells are resuspended in PBS, centrifuge sample, wash with PBS, and repeat centrifugation. (v) Remove supernatant

3.4.2 Collecting 3D Cells from ECM Method
7. Add $1 \mathrm{~mL}$ PBS and resuspend spheroids in solution.

8. Spin again.

9. Remove PBS and freeze pellet of spheroids in $-80{ }^{\circ} \mathrm{C}$ freezer for protein analysis.

10. Once $3 \mathrm{D}$ cells have been pelleted they are ready for RTK detection as would be performed with monolayer pellets using standard procedures (e.g., by immunofluorescence, ELISA, or immunoblots).

1. Remove medium/ECM solution (Fig. 2).

2. Wash wells twice with $100 \mu \mathrm{L}$ ice-cold PBS.

3. Add $150 \mu \mathrm{L}$ ice-cold PBS and lift cells in ECM with pipette tip.

4. Agitate cells in PBS for $30 \mathrm{~min}$ on ice to help break up cells.

5. Remove cells and ECM from wells with a pipette and transfer contents to a $15 \mathrm{~mL}$ centrifuge tube (see Note 8 ).

6. Continue to break up cells and ECM on ice for 15-20 min.

7. If ECM has dissolved completely centrifuge solution for $5 \mathrm{~min}$ at $900 \times g$, if not add more PBS and continue to agitate until dissolved and then centrifuge.

8. Remove supernatant and resuspend cell pellet with ice-cold PBS; repeat centrifugation. 
9. Remove PBS and freeze pellet of spheroids in $-80{ }^{\circ} \mathrm{C}$ freezer for subsequent analysis.

10. Once $3 \mathrm{D}$ cells have been pelleted they are ready for RTK detection as would be performed on monolayer pellets using standard procedures.

\section{Notes}

1. Time can be saved if 96-well plates are coated with polyHEMA solution first thing in the morning. If this is done, by evening time, the first coat of poly-HEMA should be evaporated and the second coat can be applied and allowed to evaporate overnight.

2. It is essential that poly-HEMA-coated plates are completely dry before addition of cells in culture medium. If any polyHEMA solution remains in wells of the plate when culture medium added, the poly-HEMA solution turns cloudy and renders the wells useless.

3. Seeding density described here is for HCC1954 cells and should be adjusted accordingly for individual cell lines.

4. When changing medium on cells grown in $3 \mathrm{D}$ it is important to pipette gently so as not to disturb the spheroid at the bottom of a well. Only insert the pipette tip halfway into the culture medium and when placing fresh medium back into a well, dispense the medium slowly and down the side of the wall of the well. Some cell lines do not form tightly packed spheroids when grown in 3D (e.g., SKBR3 cells); in these cases cell aggregates are formed and can easily be disrupted by fast pipetting.

5. When pipetting drops it is essential that they are exactly in the centre of the ring or else when the lid is replaced the drops can get caught on the walls of the wells and are ruined.

6. ECM gels at room temperature so must be kept chilled to ensure that it is in liquid form for plating to enable easy handling.

7. Tips are coated with $1 \%$ BSA to prevent the cell spheroids from sticking to the inside and outside of the tips during washing steps.

8. If some cells and ECM remain in wells, perform an additional washing step with ice-cold medium rinsed from wells.

\section{Acknowledgements}

Preparation of this chapter was supported by the Marie Keating Foundation; Irish Cancer Society's support of Breast-PREDICT [CCRC13GAL]; the Health Research Board [HRA_POR/2013/ 342]; and Higher Education Authority's PRTLI Cycle 5 support of TBSI. 


\section{References}

1. Harrison RG, Greenman MJ, Mall FP, Jackson CM (1907) Observations of the living developing nerve fiber. Anat Rec 1:116-128

2. Bissell MJ, Rizki A, Mian IS (2003) Tissue architecture: the ultimate regulator of breast epithelial function. Curr Opin Cell Biol 15:753-762

3. Weaver VM, Petersen OW, Wang F et al (1997) Reversion of the malignant phenotype of human breast cells in three-dimensional culture and in vivo by integrin blocking antibodies. J Cell Biol 137:231-245

4. Stahl A, Wenger A, Weber H et al (2004) Bi-directional cell contact-dependent regulation of gene expression between endothelial cells and osteoblasts in a three-dimensional spheroidal coculture model. Biochem Biophys Res Commun 322:684-692

5. Pickl M, Ries $\mathrm{CH}$ (2008) Comparison of 3D and 2D tumor models reveals enhanced HER2 activation in $3 \mathrm{D}$ associated with an increased response to trastuzumab. Oncogene 28:461-468

6. Breslin S, O'Driscoll L (2013) Threedimensional cell culture: the missing link in drug discovery. Drug Discov Today 18:240-249
7. Ivascu A, Kubbies M (2006) Rapid generation of single-tumor spheroids for high-throughput cell function and toxicity analysis. J Biomol Screen 11:922-932

8. Kelm JM, Timmins NE, Brown CJ et al (2003) Method for generation of homogeneous multicellular tumor spheroids applicable to a wide variety of cell types. Biotechnol Bioeng 83: 173-180

9. Justice BA, Badr NA, Felder RA (2009) 3D cell culture opens new dimensions in cell-based assays. Drug Discov Today 14:102-107

10. Kleinman HK, Martin GR (2005) Matrigel: basement membrane matrix with biological activity. Semin Cancer Biol 15:378-386

11. Goodwin TJ, Prewett TL, Wolf DA, Spaulding GF (1993) Reduced shear stress: a major component in the ability of mammalian tissues to form three-dimensional assemblies in simulated microgravity. J Cell Biochem 51:301-311

12. Lin RZ, Chang HY (2008) Recent advances in three-dimensional multicellular spheroid culture for biomedical research. Biotechnol J 3: $1172-1184$ 